\title{
Estrategias didácticas y comprensión lectora en educación básica
}

\section{Didactic strategies and reading comprehension in basic education}

DOI: $10.46932 / \mathrm{sfjdv} 3 \mathrm{n} 1-006$

Received in: Dec 30st, 2021

Accepted in: Jan 1th, 2022

\author{
Nora Mercedes Torres Torres \\ Magister en Bibliotecología \\ Universidad Regional Autónoma de los Andes Cuarto nivel \\ Av. Delta s/n y Av. Kennedy. \\ Universidad "César Vallejo" Piura -Perú \\ E-mail: n.torrest2309@gmail.com \\ César Balladares Atoche \\ Profesión: Licenciado en Educación \\ Grado académico: Doctor en Educación \\ Calle Tupac Amaru 326 San Jacinto - Tumbes \\ Universidad "César Vallejo" Piura -Perú \\ E-mail: cballadaresa@ucvvirtual.edu.pe
}

\section{Ana Maricela Jácome Vera}

Magister en diseño y evaluación de modelos Educativos

Universidad Tecnológica Empresarial de Guayaquil Guayaquil-Ecuador

Docente

Rectora UEF Rep. de Venezuela

Tulcán entre Argentina y San Martin

Universidad "César Vallejo" Piura -Perú

E-mail: maricela_jacome73@ hotmail.com

\section{Betty Salavarria Barco}

Magíster en diseño y Evaluación de Modelos Educativos

Universidad Tecnológica Empresarial de Guayaquil, Guayaquil-Ecuador

Docente nivel primario

Cantón Salitre

Universidad "César Vallejo" Piura -Perú

E-mail: bettysalavarria@hotmail.com

\section{RESUMEN}

La presente tesis denominada: Estrategias didácticas y la comprensión lectora en estudiantes de una Unidad Educativa Fiscal de la Provincia del Guayas "., tuvo como objetivo el determinar la relación que existe entre las estrategias didácticas y la comprensión lectora en los estudiantes, fue de tipo no experimental, asumió el diseño correlacional causal, de enfoque cuantitativo, tomó en cuenta una población de 270 estudiantes y 159 en la muestra, aplicó la técnica de la encuesta y empleó como instrumentos dos cuestionarios, investigación que obtuvo como resultados que el 88,1\% de los estudiantes se ubicaron en el nivel medio para la comprensión lectora y otros 19 estudiantes que representan el 11,9\% lograron un nivel bajo en comprensión lectora, llegando a la conclusión que las estrategias didácticas se 
relacionan con la comprensión lectora de los estudiantes, debido a que el 76,1\% de los estudiantes que trabajaba con estrategias didácticas, mejoran su comprensión lectora, pues el 88,1\% de los estudiantes lograron un nivel medio, relación que se comprueba con el valor de Rho de Spearman obtenido de 0,790 que indicó una correlación positiva alta, con un Sig. (bilateral) de 0,000, valor significativo en función de alfa de 0,01 bilateral.

Palabras clave: Estrategia, comprensión, didáctica, lectura.

\begin{abstract}
The present thesis called: Didactic strategies and reading comprehension in students of a Fiscal Educational Unit of the Province of Guayas ". Its objective was to determine the relationship that exists between didactic strategies and reading comprehension in students. non-experimental, assumed the causal correlational design, with a quantitative approach, took into account a population of 270 students and 159 in the sample, applied the survey technique and used two questionnaires as instruments, research that obtained as results that $88.1 \%$ of the students were located at the medium level for reading comprehension and another 19 students representing $11.9 \%$ achieved a low level in reading comprehension, concluding that the didactic strategies are related to the reading comprehension of the students., due to the fact that $76.1 \%$ of the students who worked with didactic strategies improved their reading comprehension, since $88,1 \%$ of the students achieved a medium level, a relationship that is verified with the Spearman Rho value obtained of 0.790 , which indicated a high positive correlation, with a Sig. (Bilateral) of 0.000, significant value as a function of alpha of 0,01 bilateral.
\end{abstract}

Keywords: Strategy, comprehension, didactics, reading.

\title{
1 INTRODUCCIÓN
}

El nivel de logro de los estudiantes en las diferentes áreas o asignaturas se viene midiendo a través de diversos instrumentos o aplicativos a nivel mundial y sobre todo en las áreas consideradas principales, dentro de ellas la lectura, cuyos resultados causan preocupación pues la lectura y la comprensión nos sirven en la formación y nos brindan habilidad para desenvolverse adecuadamente.

En Colombia, país donde se viene mejorando en lectura, aún presenta inconvenientes para desarrollar las capacidades mínimas, pues de acuerdo a los resultados obtenidos en la prueba, alcanzó en lectura el 51,0\% de educandos mejoraron el nivel mínimo llamado nivel 2, nada prometedor, pues existe un 49,0\% de estudiantes que aún no superan el nivel mínimo, en cuanto a la representación del significado explícito en el texto, como para la integración de la información para generar inferencias, se logró un puntaje en promedio de 413, que en comparación al máximo obtenido de 487 puntos evidencia un promedio bajo, de igual manera para la capacidad para evaluar lo referido a la calidad o la credibilidad de la información llegando a reflexionar en el contenido como de la forma del texto, se obtuvo 417 puntos, que igual se encuentra por debajo de los 489 puntos del máximo obtenido, que indica deficiencias en la comprensión de textos que implica trabajar estrategias innovadoras para la mejora (PISA, Icfes, 2020). 
En Ecuador, en cuanto a los resultados del mismo año que fuera evaluado Colombia, de acuerdo a MINEDUC (2018) que expresa los resultados PISA correspondientes al año 2018, indica que, en el caso de lectura, se logró en promedio de 409, que a pesar de ser promedio alto alcanzado para dicho año, se debe indicar que el promedio mayor alcanzado fue de 496 puntos, debiendo precisar que contaba con estudiantes por debajo del nivel básico referido a la capacidad de lectura, ello debido a que fue el 51,0\% de los educandos, los que no lograron el nivel 2, y por otro lado se observa que el 38,0\%, se encuentran en el nivel 1a; quienes pueden localizar ya sea uno como más fragmentos independientes de información que se encuentran explícitos, además de identificar el tema ya sea principal como el propósito que tienen el autor del texto, luego se alcanza a observar que se cuenta con estudiantes cuyo desempeño se encuentra por debajo del nivel $1^{a}$, los que lograron el nivel 1 b fueron el 15,5\%, ello indica que solo son capaces de resolver asignaciones de comprensión lectora básica, limitado a buscar un único dato señalado específicamente, en el título del texto sencillo como que pertenezca a una lista sencilla.

El Problema expuesto anteriormente a nivel nacional, en cuanto al nivel de logro de las competencias para la comprensión lectora es común a nivel local, pues los estudiantes presentan inconvenientes para comprender lo que leen, desempeño que no se evidencia en casi todas las Unidades Educativas, y en especial de la Unidad Educativa Fiscal de la provincia del Guayas, sobre todo en los estudiantes que cursan el 8vo de educación básica, bajo nivel de logro que nos permitió formular el siguiente problema: ¿Cuál es la relación que existe entre las estrategias didácticas y la comprensión lectora en los estudiantes de 8vo de básica en la Unidad Educativa Fiscal de la provincia del Guayas.

El objetivo general, quedó compuesto de la siguiente manera: Determinar la relación que existe entre las estrategias didácticas y la comprensión lectora en los estudiantes de 8vo de básica en la Unidad Educativa de la provincia del Guayas.

El estudio se justifica, en cuanto a su relevancia práctica, en el sentido, que el ser humano por ser sociable, siente la necesidad de comunicarse con los demás, interpretar los mensajes y sobre todo comprenderlos, es cuando adquiere las capacidades para comprender lo que se requiere que se convierte en un ser competente en los diferentes ámbitos, tanto familiares, sociales e institucionales, pues para destacar en el mundo laboral tendrá que saber recibir el mensaje, interpretarlo e implementarlo conforme lo que se requiera.

En ese sentido, se procedió a formular la hipótesis general: H1: Las estrategias didácticas se relacionan con la comprensión lectora en los estudiantes de 8vo de básica en la Unidad Educativa Fiscal de la provincia del Guayas. Y entre las hipótesis específicas HE1: Las estrategias didácticas se relacionan con el nivel literal de la comprensión lectora en los estudiantes. HE2: Las estrategias didácticas se relacionan con el nivel inferencial de la comprensión lectora en los estudiantes. HE3: Las estrategias didácticas se relacionan con el nivel crítico de la comprensión lectora en los estudiantes. 


\section{METODOLOGÍA}

CONCYTEC (2018) establece dos tipos de investigación, la pura y aplicada; expresa que la investigación aplicada está orientada a determinar conclusiones haciendo uso del conocimiento científico y medios como: metodología, protocolos y tecnologías a través de las cuales se llega a la solución de una necesidad.

El diseño viene hacer el plan a tomar en cuenta para la ejecución de la investigación. Hernández, et al. (2014), indica lo siguiente:

Los diseños Transeccionales, llamados transversales, tienen como propósito el recoger información durante un solo momento, es decir, en un mismo tiempo, se requiere describir las variables de estudio y analizar cómo es que se presenta el grado de incidencia de una variable sobre la otra durante un momento dado, algunos la interpretan como aquella fotografía del momento. (p. 154).

El diseño utilizado fue el correlacional causal, buscó encontrar la relación entre las dos variables, tomando en cuenta la causa y efecto.

\section{RESULTADOS}

Del primer objetivo específico: Identificar la relación entre las estrategias didácticas y el nivel literal de la comprensión lectora, en los estudiantes.

Tabla 01. Relación entre estrategias didácticas y nivel literal de la comprensión lectora.

\begin{tabular}{rccccc}
\hline & & \multicolumn{2}{c}{ Nivel literal } & \multirow{2}{*}{ Total } \\
& & & Bajo & Medio & \multicolumn{1}{c}{ ( } \\
& \multirow{3}{*}{ A veces } & Recuento & 21 & 17 & 38 \\
Estrategias didácticas & & $\%$ & $13,2 \%$ & $10,7 \%$ & $23,9 \%$ \\
& \multirow{2}{*}{ Siempre } & Recuento & 15 & 106 & 121 \\
& & $\%$ & $9,4 \%$ & $66,7 \%$ & $76,1 \%$ \\
& & Recuento & 36 & 123 & 159 \\
& & $\%$ & $22,6 \%$ & $77,4 \%$ & $100,0 \%$ \\
\hline
\end{tabular}

Nota: Cuestionario para estudiantes

Figura 01. Relación entre estrategias didácticas y nivel literal de la comprensión lectora.

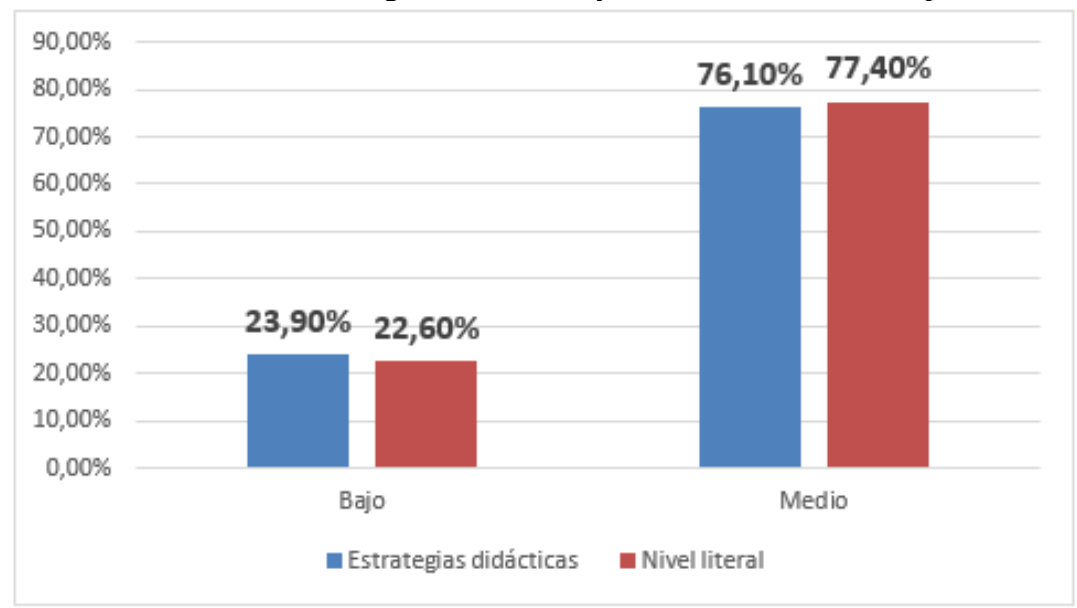


Interpretación: La tabla nos muestra los resultados de la relación que existe entre las estrategias didácticas con el nivel literal de la comprensión lectora, donde fueron 121 estudiantes que representan el $76,1 \%$ los que siempre trabajan estrategias didácticas, mientras que 123 estudiantes que representan el $77,4 \%$ los que se ubican en un nivel literal medio de la comprensión lectora, debiendo indicar que son 38 estudiantes que vienen hacer el 23,9\% los que a veces trabajan con estrategias didácticas y 36 estudiantes que representan el 22,6\% los que cuentan con un nivel literal bajo en la comprensión lectora, que se deben tomar en cuenta como necesidad de aprendizaje a mejorar.

Del segundo objetivo específico: Estimar la relación entre las estrategias didácticas y el nivel inferencial de la comprensión lectora, en los estudiantes.

Tabla 02. Relación entre las estrategias didácticas y nivel inferencial de la comprensión lectora.

\begin{tabular}{ccccccc}
\hline & & \multicolumn{3}{c}{ Nivel inferencial } & \multicolumn{2}{c}{ Total } \\
& & & Bajo & Medio & Alto & \\
\hline & A veces & Recuento & 18 & 20 & 0 & 38 \\
Estrategias didácticas & & $\%$ & $11,3 \%$ & $12,6 \%$ & $0,0 \%$ & $23,9 \%$ \\
& Siempre & Recuento & 3 & 108 & 10 & 121 \\
& & $\%$ & $1,9 \%$ & $67,9 \%$ & $6,3 \%$ & $76,1 \%$ \\
& & Recuento & 21 & 128 & 10 & 159 \\
& & $\%$ & $13,2 \%$ & $80,5 \%$ & $6,3 \%$ & $100,0 \%$ \\
\hline
\end{tabular}

Nota: Cuestionario para estudiantes

Figura 02. Relación entre las estrategias didácticas y nivel inferencial de la comprensión lectora.

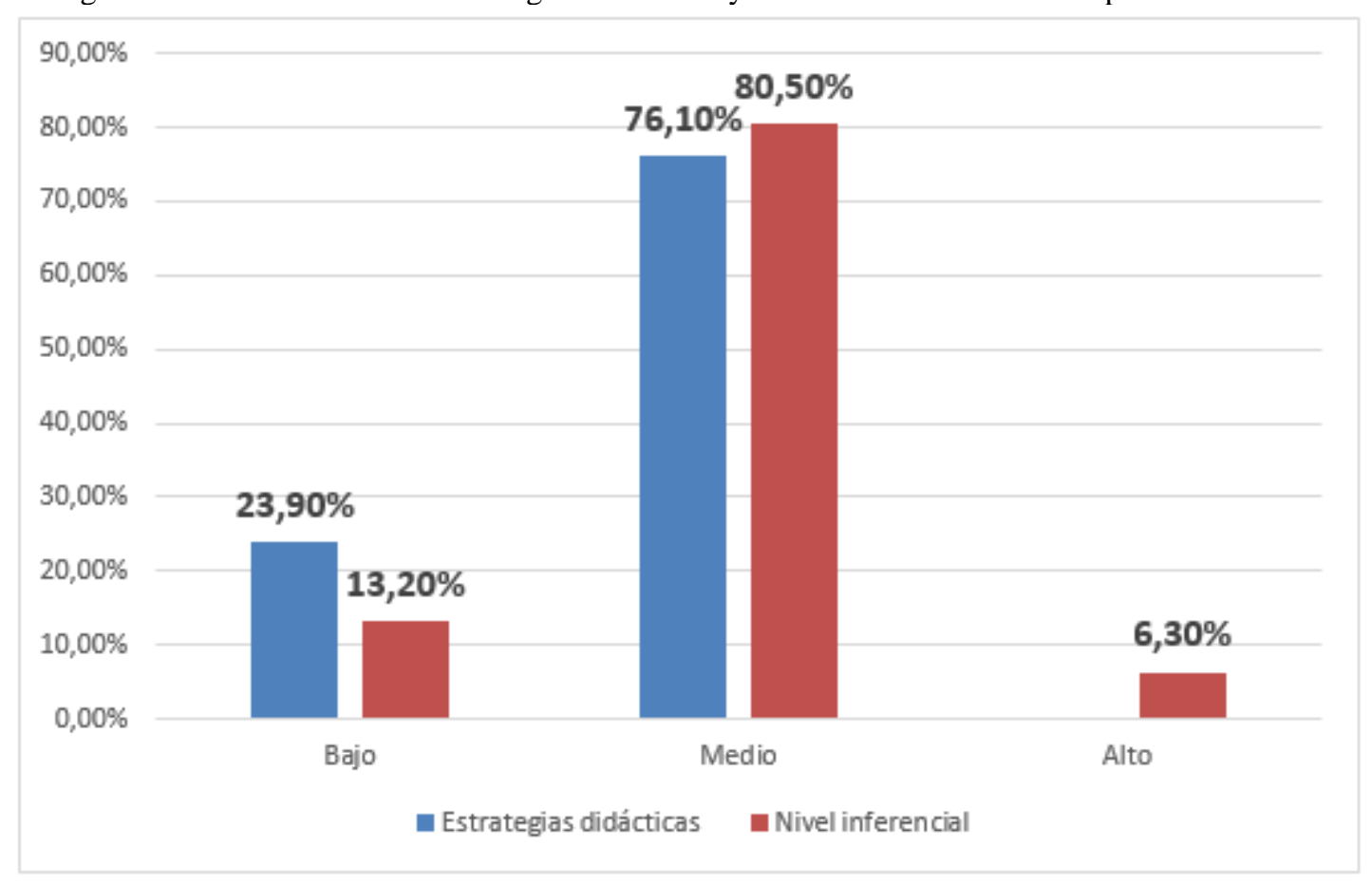


Interpretación: La tabla nos muestra los resultados de la relación que existe entre la primera variable de las estrategias didácticas con la dimensión del nivel inferencial de la comprensión lectora, donde fueron 121 estudiantes que equivalen al 76,1\% los que siempre trabajan con estrategias didácticas y por otro lado 128 estudiantes que representan el 80,5\% los que se ubicaron en el nivel inferencial medio de la comprensión lectora, debiendo tomar en cuenta que 21 estudiantes que representan el 13,2\% se ubican en el nivel inferencial bajo en comprensión lectora y sobre los cuales se debe trabajar, sin embargo fueron 10 estudiantes que representan el 6,3\% los que alcanzaron un nivel inferencial alto para la comprensión lectora.

Del tercer objetivo específico: Establecer la relación entre las estrategias didácticas y el nivel crítico de la comprensión lectora, en estudiantes.

Tabla 03. Relación entre las estrategias didácticas y el nivel crítico de la comprensión lectora.

\begin{tabular}{ccccccc}
\hline & & \multicolumn{3}{c}{ Nivel critico } & \multicolumn{2}{c}{ Total } \\
& & & Bajo & Medio & Alto & \\
\hline & A veces & Recuento & 13 & 25 & 0 & 38 \\
Estrategias didácticas & & $\%$ & $8,2 \%$ & $15,7 \%$ & $0,0 \%$ & $23,9 \%$ \\
& Siempre & Recuento & 2 & 109 & 10 & 121 \\
& & $\%$ & $1,3 \%$ & $68,6 \%$ & $6,3 \%$ & $76,1 \%$ \\
& & Recuento & 15 & 134 & 10 & 159 \\
& & $\%$ & $9,4 \%$ & $84,3 \%$ & $6,3 \%$ & $100,0 \%$ \\
\hline
\end{tabular}

Nota: Cuestionario para estudiantes

Figura 03. Relación entre las estrategias didácticas y nivel crítico de la comprensión lectora.

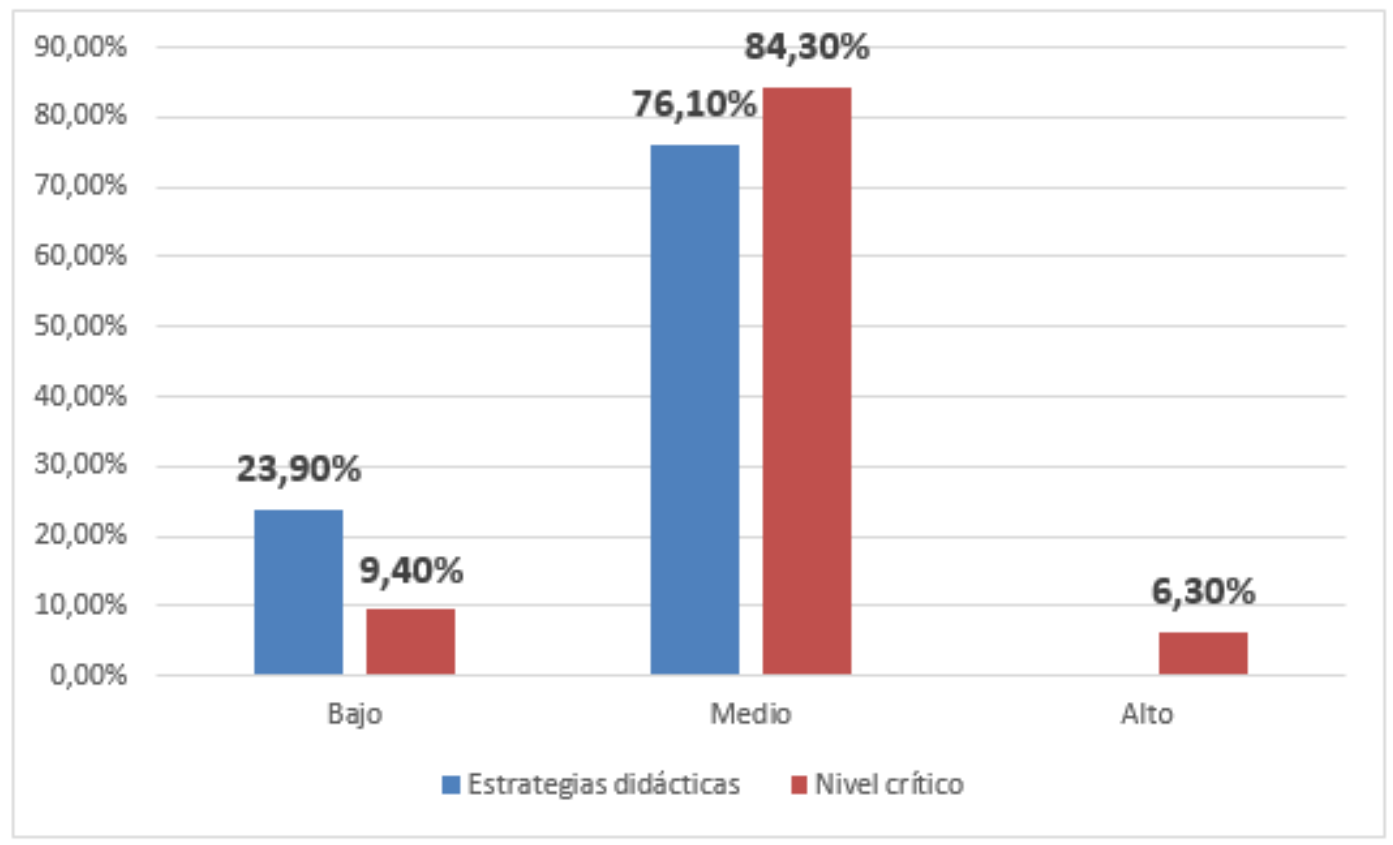


Interpretación: La tabla nos muestra los resultados de la relación que existe entre la variable estrategias didácticas con la dimensión del nivel crítico de la comprensión lectora, donde fueron 121 estudiantes que representan el 76,1\% los que siempre trabajan estrategias didácticas, y por otro lado fueron 134 estudiantes que representan el 84,3\% los que se ubicaron en un nivel medio de la comprensión lectora, debiendo tomar en cuenta que 38 estudiantes que equivalen al 23,9\% los que a veces trabajan con estrategias didácticas y 15 estudiantes que representan el 9,4\% los que se ubicaron en el nivel bajo de la comprensión lectora, siendo una necesidad para la mejora en el aprendizaje de los estudiantes, mientras que fueron 10 estudiantes que representan el 6,3\% los que alcanzaron un nivel crítico alto para la comprensión lectora.

Del objetivo general: Determinar la relación que existe entre las estrategias didácticas y la comprensión lectora en los estudiantes de 8vo de básica de la provincia del Guayas

Tabla 04. Relación entre las estrategias didácticas y la comprensión lectora.

\begin{tabular}{cccccc}
\hline & & & \multicolumn{2}{c}{ Comprensión lectora } & Total \\
& & & Bajo & Medio & \\
\hline & A veces & Recuento & 17 & 21 & 38 \\
Estrategias didácticas & & $\%$ & $10,7 \%$ & $13,2 \%$ & $23,9 \%$ \\
& \multirow{2}{*}{ Siempre } & Recuento & 2 & 119 & 121 \\
& & $\%$ & $1,3 \%$ & $74,8 \%$ & $76,1 \%$ \\
& & Recuento & 19 & 140 & 159 \\
& & $\%$ & $11,9 \%$ & $88,1 \%$ & $100,0 \%$ \\
\hline
\end{tabular}

Nota: Cuestionario para estudiantes

Figura 04. Relación entre las estrategias didácticas y la comprensión lectora.

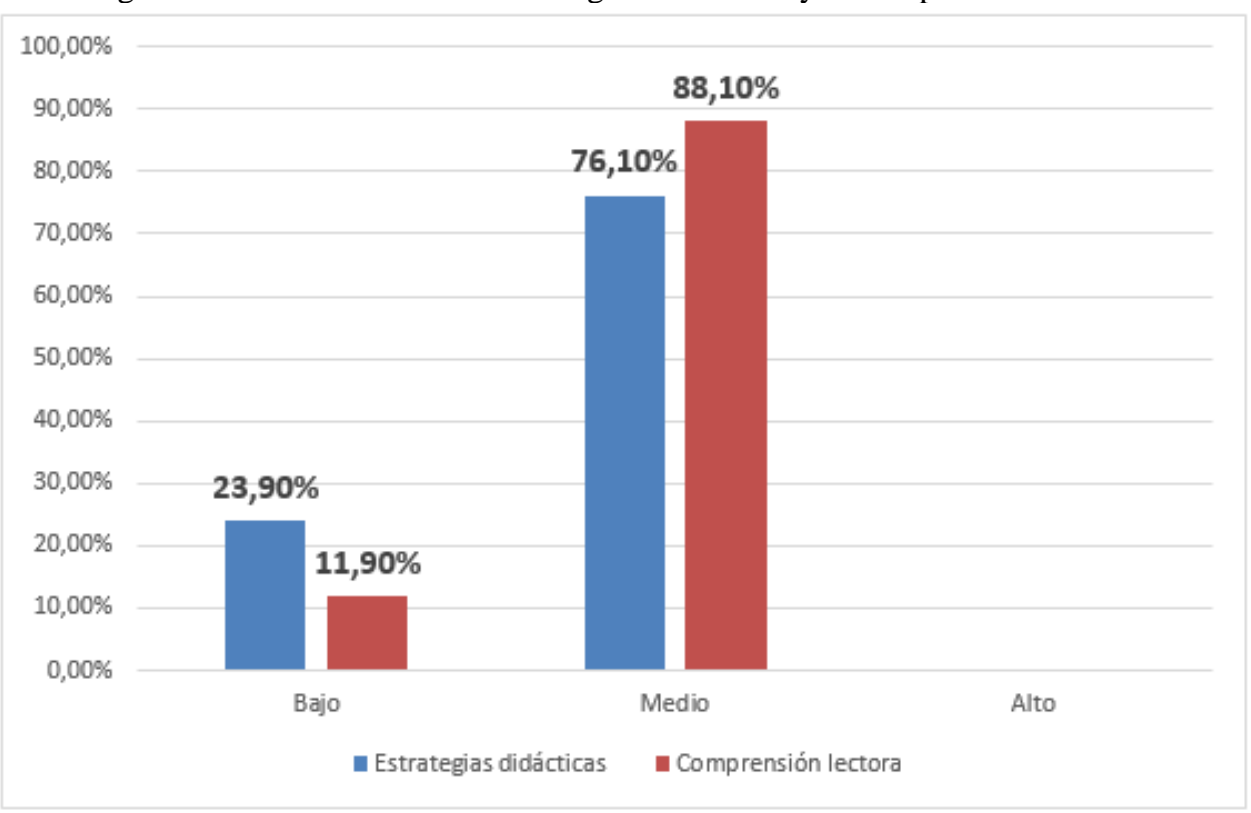


Interpretación: La tabla nos muestra la relación que existe entre las dos variables del estudio, donde de los 159 estudiantes evaluados, fueron 121 de ellos que vienen hacer el 76,1\% los que siempre trabajan con estrategias didácticas, sin embargo fueron 140 estudiantes que representan el $88,1 \%$ los que se ubicaron en el nivel medio para la comprensión lectora y otros 19 estudiantes que representan el 11,9\% los que lograron un nivel bajo en comprensión lectora, que son los estudiantes con necesidades y sobre los cuales se debe trabajar para la mejora de su aprendizaje.

Para la comprobación de las hipótesis

HE1: Las estrategias didácticas se relacionan con el nivel literal de la comprensión lectora en los estudiantes.

Tabla 05. Correlación entre las estrategias didácticas y el nivel literal de la comprensión lectora.

\begin{tabular}{ccccc}
\hline & & & $\begin{array}{c}\text { Estrategias } \\
\text { didácticas }\end{array}$ & Nivel literal \\
& Estrategias & Coeficiente de correlación & 1,000 &, $437^{* *}$ \\
& didácticas & Sig. (bilateral) &. &, 000 \\
Rho de & & $\mathrm{N}$ & 159 & 159 \\
Spearman & Nivel literal & Coeficiente de correlación &, $437^{* *}$ & 1,000 \\
& & Sig. (bilateral) &, 000 &. \\
& & $\mathrm{~N}$ & 159 & 159 \\
\hline
\end{tabular}

**. La correlación es significativa en el nivel 0,01 (bilateral).

Interpretación: La tabla nos muestra la correlación que existe entre la variable de las estrategias didácticas con la dimensión del nivel literal de la comprensión lectora, donde el valor de Rho de Spearman encontrado de 0,437 nos indica la existencia de una correlación positiva moderada, además que el Sig. (bilateral) encontrado de 0,000, evidencia la significatividad por ser un valor menor que el valor de alfa de 0,01 bilateral, por consiguiente se procedió con la aprobación de la hipótesis alternativa.

HE2: Las estrategias didácticas se relacionan con el nivel inferencial de la comprensión lectora en los estudiantes.

Tabla 06. Correlación entre las estrategias didácticas y el nivel inferencial de la comprensión lectora.

\begin{tabular}{|c|c|c|c|c|}
\hline & & & $\begin{array}{l}\text { Estrategias } \\
\text { didácticas }\end{array}$ & Nivel inferencial \\
\hline \multirow{6}{*}{ Rho de Spearman } & \multirow{3}{*}{$\begin{array}{l}\text { Estrategias } \\
\text { didácticas }\end{array}$} & Coeficiente de correlación & 1,000 &, $650^{*+}$ \\
\hline & & Sig. (bilateral) & . &, 000 \\
\hline & & $\mathrm{N}$ & 159 & 159 \\
\hline & \multirow{3}{*}{ Nivel inferencial } & Coeficiente de correlación &, $650^{* *}$ & 1,000 \\
\hline & & Sig. (bilateral) &, 000 & . \\
\hline & & $\mathrm{N}$ & 159 & 159 \\
\hline
\end{tabular}

**. La correlación es significativa en el nivel 0,01 (bilateral). 
Interpretación: La tabla nos muestra la correlación que existe entre la variable de las estrategias didácticas con la dimensión del nivel inferencial de la comprensión lectora, donde el valor de Rho de Spearman encontrado de 0,650 nos indica la existencia de una correlación positiva moderada, además que el Sig. (bilateral) encontrado de 0,000, evidencia la significatividad por ser un valor menor que el valor de alfa de 0,01 bilateral, por consiguiente se procedió con la aprobación de la hipótesis alternativa.

HE3: Las estrategias didácticas se relacionan con el nivel crítico de la comprensión lectora en los estudiantes.

Tabla 07. Correlación entre las estrategias didácticas y el nivel crítico de la comprensión lectora.

\begin{tabular}{ccccc}
\hline & & & $\begin{array}{c}\text { Estrategias } \\
\text { didácticas }\end{array}$ & Nivel crítico \\
& Estrategias & Coeficiente de correlación & 1,000 &, $694^{* *}$ \\
& didácticas & Sig. (bilateral) & $\cdot$ &, 000 \\
Rho de & & $\mathrm{N}$ & 159 & 159 \\
Spearman & Nivel crítico & Coeficiente de correlación &, $694^{* *}$ & 1,000 \\
& & Sig. (bilateral) &, 000 &. \\
& & $\mathrm{~N}$ & 159 & 159 \\
\hline
\end{tabular}

**. La correlación es significativa en el nivel 0,01 (bilateral).

Interpretación: La tabla nos muestra la correlación que existe entre la variable de las estrategias didácticas con la dimensión del nivel crítico de la comprensión lectora, donde el valor de Rho de Spearman encontrado de 0,694 nos indica la existencia de una correlación positiva moderada, además que el Sig. (bilateral) encontrado de 0,000, evidencia la significatividad por ser un valor menor que el valor de alfa de 0,01 bilateral, por consiguiente se procedió con la aprobación de la hipótesis alternativa.

Hipótesis general: H1: Las estrategias didácticas se relacionan con la comprensión lectora en los estudiantes de 8vo de básica en la Unidad Educativa Fiscal de la provincia del Guayas

Tabla 08. Correlación entre las estrategias didácticas y la comprensión lectora.

\begin{tabular}{lcccc}
\hline & & & $\begin{array}{c}\text { Estrategias } \\
\text { didácticas }\end{array}$ & $\begin{array}{c}\text { Comprensión } \\
\text { lectora }\end{array}$ \\
\hline \multirow{2}{*}{ Rho de Spearman } & Estrategias & Coeficiente de correlación & 1,000 &, $790^{* *}$ \\
& didácticas & Sig. (bilateral) &. &, 000 \\
& Comprensión & Coeficiente de correlación &, $790^{* *}$ & 159 \\
& lectora & Sig. (bilateral) &, 000 & 1,000 \\
& & $\mathrm{~N}$ & 159 & 159 \\
\hline
\end{tabular}

**. La correlación es significativa en el nivel 0,01 (bilateral). 
Interpretación: La tabla nos muestra la correlación que existe entre la variable de las estrategias didácticas con la variable de la comprensión lectora, donde el valor de Rho de Spearman encontrado de 0,790 nos indica la existencia de una correlación positiva alta, además que el Sig. (bilateral) encontrado de 0,000 , evidencia la significatividad por ser un valor menor que el valor de alfa de 0,01 bilateral, por consiguiente, se procedió con la aprobación de la hipótesis general y rechazo de la hipótesis nula.

\section{DISCUSIÓN}

Respecto al objetivo general para determinar la relación que existe entre las estrategias didácticas y la comprensión lectora en los estudiantes de 8vo de básica en la Unidad Educativa Fiscal de la provincia del Guayas.

Dentro de los hallazgos encontrados en los estudiantes respecto a la comprensión lectora, las podemos dimensionar en tres aspectos, primero la complejidad que se evidencia para obtener la información del texto que lee el estudiante, donde aún no se le hace fácil el localizar como para seleccionar la información incluso que se encuentra explícita, luego cuando tienen que inferir como para interpreta información de la lectura, no llegando a la construcción del sentido de lo que lee, el tener que inferir llegando a establecer varias relaciones hablando de lo que se encuentra explícito como implícito con el propósito de llegar a deducir información nueva, pues se trata de que logre interpretar la información explícita e implícita y finalmente los estudiantes no reflexionan antes de evaluar no solo la forma, sino también el contenido y contexto de lo que leen.

Los resultados en el estudio indicaron que el 76,1\% de los estudiantes trabajan estrategias didácticas para generar la lectura, pero el 88,1\% de ellos, todavía presenta dificultades para la comprensión pues se ubicaron en el nivel medio y por otro lado el 11,9\% apenas llegaron al nivel bajo de la comprensión lectora.

Los resultados coinciden con los encontrados por Ricaldi (2019), estudio realizado en Perú quien midió el nivel de la comprensión lectora en 576 estudiantes correspondientes al segundo grado en secundaria, cuyos resultados arrojaron que el 86.7\%, de los estudiantes se ubicaron en el nivel de inicio para el nivel de logro en la comprensión lectora que coincide con el 88,1\% que presentan dificultades para la comprensión lectora, siendo el $13.3 \%$ de los estudiantes que aún se encontraban en proceso, y de hecho ningún estudiante había logrado el nivel esperado, estudio que concuerda con la investigación realizada en cuanto a los resultados en función de porcentajes sobre la misma variable de la comprensión lectora.

En cuanto al primer objetivo específico para identificar la relación entre las estrategias didácticas y el nivel literal de la comprensión lectora, en los estudiantes. 
De los hallazgos encontrados en el estudio, se pueden mencionar que la mayoría de estudiantes presentó inconvenientes para distinguir entre la información relevante y la información secundaria del texto, del mismo modo para encontrar la idea o ideas principales, como de los personajes principales, incluso para identificar las relaciones presentes en el texto de causa-efecto, para distinguir entre las buenas y malas acciones de un personaje, cuando debía ordenar de forma secuencial las acciones que realizaba un personaje, así también para identificar la secuencia narrativa, cuando el pasado se mezclaba con el presente, se complicaba para la comprensión y finalmente para identificar los hechos más resaltantes en cada parte de una secuencia narrativa.

Los resultados del estudio indicaron que a pesar de que el 76,1\% de los estudiantes trabajaba estrategias didácticas para promover la lectura, fue el 77,4\% de ellos que se ubicaba en el nivel medio para la comprensión literal de los textos, por otro lado, el 22,6\% de los estudiantes, se ubicó en el nivel bajo para el nivel literal de la comprensión lectora, lo que desde luego debía considerarse en la propuesta para la mejora del aprendizaje lector.

Uno de los estudios coinciden en cuanto a resultados es el realizado por Guevara (2019), desarrollado en el Perú, donde de los 76 estudiantes que formaban parte de la muestra, se encontró después de la aplicación de una evaluación en lectura, que el 21,0\% de los estudiantes, se ubicaron en el nivel de inicio, y el otro 79,0\%, alcanzaron apenas ubicarse en el nivel de proceso en cuanto a las destrezas para el logro de la comprensión lectora sobre todo para lograr el nivel literal que coincide con el 77,4\% de los estudiantes que en el estudio se ubicaron en el nivel medio para la comprensión literal de la lectura, es decir que los resultados concuerdan comparando la escala de medición tanto para el nivel medio como para el de proceso de la comprensión que se constituye en el nivel básico de la lectura y su entendimiento.

Para el segundo objetivo específico de estimar la relación entre las estrategias didácticas y el nivel inferencial de la comprensión lectora, en los estudiantes.

Los hallazgos encontrados para lograr el nivel inferencial en los estudiantes fueron que la mayoría al momento de observar el título como la portada de un texto no lograba inferir de qué se trataba la lectura, por otro lado, el tener que inferir las consecuencias de los hechos presentados en el texto, para tener que predecir lo que pasaría con los personajes, cuando se le solicitaba el interpretar las frases desarrolladas pero con lenguaje metafórico, cuando tenía que interpretar de manera correcta el lenguaje figurado, incluso que si el texto presentaba palabras sencillas, no comprendía lo que leía, en cuanto al manejo del diccionario para las palabras desconocidas, debido a que no llegaba a inferir el significado de las palabras que no conocía y para tener que deducir el significado de cualquier palabra, tomando en cuenta el contexto del cual formaba parte.

Los resultados del estudio indicaron que, en cuanto al nivel inferencial, fue el $80,5 \%$ de los estudiantes que se encontraban ubicados en el nivel medio para el logro del nivel inferencial del proceso 
de la comprensión lectora, sin dejar de lado la mayor necesidad, debido a que el 13,2\% de los estudiantes, se ubicaron en el nivel bajo para el logro del nivel inferencial de la comprensión lectora, para con quienes se debe trabajar para la mejora de dichas capacidades.

Uno de los estudios que coinciden en cuanto a los resultados es el de Arapa (2019), quien, al evaluar a 40 estudiantes, sujetos de la muestra, el 95,0\% de los estudiantes, alcanzaron solo ubicarse en el nivel regular para la comprensión lectora especialmente para alcanzar el nivel inferencial de la lectura y que coinciden con el 80,5\% obtenido en la investigación para el nivel medio comparando el nivel de equivalencia de la valoración, siendo el otro 5\% de estudiantes que alcanzara el nivel bueno, lo que generó preocupación en el mencionado estudio, estudio que concuerda en función de los resultados al ubicarse el mayor porcentaje tanto en el nivel medio como para el regular.

Finalmente, para el tercer objetivo específico de establecer la relación entre las estrategias didácticas y el nivel crítico de la comprensión lectora, en estudiantes.

En cuanto al nivel crítico, los hallazgos fueron que los estudiantes presentaban problemas para con facilidad poder emitir sus opiniones de tipo personal, por supuesto en relación al texto leído, otra dificultad encontrada fue para contar con la capacidad de realizar críticas respecto a lo que lee, y cuando se trabaja en grupo, difícilmente, comparte sus opiniones con los que le rodean, otro aspecto fue al momento de tener que dar una opinión referida al comportamiento de alguno de los personajes encontrados en el texto, luego para poder elaborar una opinión de carácter personal tomando en cuenta los hechos, como para llegar a cuestionarlos y validarlos después de la lectura, siendo el aspecto más relevante la facilidad que deberían tener para identificar la intención del autor, y al momento de indagar sobre si la lectura aportaba para el desarrollo de su vida y finalmente para saber que si la lectura de alguna manera influye para el cambio de la actitud.

Los resultados para el nivel crítico indicaron que el 84,3\% de los estudiantes que formaban parte de la muestra de estudio, fueron los que llegaron a ubicarse en el nivel medio para llegar al nivel crítico de la comprensión lectora, por otra parte, el 9,4\% de los estudiantes se ubicaron en el nivel bajo de la comprensión lectora, para el nivel crítico.

Fueron los resultados de Molina (2016), que se relacionan con los encontrados en el estudio, pues al evaluar a 141 estudiantes, fueron el $35 \%$ de estudiantes, los que buscaban las ideas referidas al tema, por otro lado el $29 \%$ de estudiantes utilizaba la observación de imágenes, que les orientaba a reconocer el tipo de texto, así como para cuando se tenía que sacar las ideas en la lectura, para posteriormente tener que hacer predicciones, cabe indicar, que solo el $27 \%$ de los estudiantes llegó a reconocer el tipo de texto seleccionado para el proceso de la lectura, resultados que coinciden en cuanto el menor porcentaje se encontraba en el nivel bajo para la comprensión critica de la lectura en ambos estudios. 


\section{CONCLUSIONES}

Las estrategias didácticas se relacionan con la comprensión lectora de los estudiantes de 8vo de básica en la Unidad Educativa Fiscal de la Provincia del Guayas, conforme se deduce de los resultados que indicaron que el $76,1 \%$ de los estudiantes que trabajaba con estrategias didácticas, mejoraron la comprensión lectora, pues el 88,1\% de los estudiantes lograron un nivel medio, relación que se comprueba con el valor de Rho de Spearman obtenido de 0,790 que indicó una correlación positiva alta, con un Sig. (bilateral) de 0,000, valor significativo en función de alfa de 0,01 bilateral.

as estrategias didácticas se relacionan con el nivel literal de la comprensión lectora de los estudiantes, conforme se infiere en los resultados que indican que el 76,1\% de los estudiantes que trabajaban estrategias didácticas en las sesiones de aprendizaje, del mismo modo el 77,4\% de ellos lograron un nivel literal medio para la comprensión lectora, de donde se deduce que cuanto más se trabajen estrategias didácticas en las sesiones del área de comunicación, se alcanzarán mejores logros de aprendizaje en el nivel literal de la comprensión lectora.

Las estrategias didácticas se relacionan con el nivel inferencial de la comprensión lectora de los estudiantes, conforme se deduce de los resultados del estudio que comprobaron que el 76,1\% de los estudiantes que siempre trabajaron estrategias didácticas, del mismo modo el 80,5\% de ellos lograron un nivel inferencial medio en la comprensión lectora, lo que permite inferir que las estrategias didácticas ayudan a mejorar la comprensión lectora de los estudiantes.

Las estrategias didácticas se relacionan con el nivel crítico de la comprensión lectora de los estudiantes, conforme se deduce de los resultados, pues cuando mejores estrategias didácticas se trabajen, mejorará el nivel de la comprensión lectora, acorde a los resultados que indicaron que el 76,1\% de los estudiantes aplican estrategias didácticas y del mismo modo el 84,3\% de estudiantes lograron un nivel medio en la comprensión lectora. 


\section{REFERENCIAS}

Aguilar, A. (2018). El plan lector "Manejo Creativo de la Lectura" para la comprensión lectora de los estudiantes de un Centro de Educación Básica Alternativa, Chulucanas 2017. (Tesis de doctorado). Universidad César Vallejo. https://repositorio.ucv.edu.pe/bitstream/handle/20.500.12692/28860/Aguilar_SA.pdf?sequence=1\&isAll owed=y

Arapa, V. (2019). Estrategias De Lectura Para Mejorar La Comprensión Lectora En Estudiantes Del Cuarto Y Quinto Año De Secundaria En Una Institución Educativa, Arequipa 2018. (Tesis doctoral). Universidad César Vallejo. https://repositorio.ucv.edu.pe/bitstream/handle/20.500.12692/37776/arapa_mv.pdf?sequence=1\&isAllo wed $=y$

BBC News Mundo. (2019). Pruebas PISA: qué dice de la educación en América Latina los malos resultados obtenidos por los países de la región. https://www.bbc.com/mundo/noticias-america-latina50685470

Espinoza, M. R. (2018). La Comprensión Lectora Y El Rendimiento Académico De Los Estudiantes De Quinto Año De Egb, De La Academia Aeronáutica "Mayor Pedro Traversari" De La Provincia De Pichincha, Cantón Quito, Año Lectivo 2016- 2017”. http://www.dspace.uce.edu.ec/bitstream/25000/14174/1/T-UCE-0010-PEO030-2018.pdf

Fonseca, L., Migliardo, G., Simian, M., Olmos, R., and León, J. (2019). Strategies to improve reading comprehension: Impact of an intervention program in Spanish. Educational Psychology, 25 (2), 91-99. https://doi.org/10.5093/psed2019a1

Guevara, S. M. (2019). Organizadores gráficos para mejorar la comprensión lectora de los estudiantes de quinto grado de educación secundaria, Santiago de Chuco 2017. (Tesis de doctorado). Universidad César Vallejo. https://repositorio.ucv.edu.pe/bitstream/handle/20.500.12692/32718/guevara_cs.pdf?sequence=4\&isAll owed $=\mathrm{y}$

Hernández, R., Fernández, C., y Baptista, P. (2014). Metodología de la Investigación, Sexta edición, México. http://docs.wixstatic.com/ugd/986864_5bcd4bbbf3d84e8184d6e10eecea8fa3.pdf

Hernández, J., and Sanabria, K. M. (2018). Meta-cognitive strategies: Scaffolding of reading comprehension. The Third Shore. https://revistas.unab.edu.co/index.php/laterceraorilla/article/view/3475 Icfes (2020). Informe Nacional de Resultados para Colombia - PISA 2018. Instituto Colombiano para la Evaluación de la Educación. https://www.icfes.gov.co/documents/20143/1529295/Informe\%20nacional\%20de\%20resultados\%20PIS A\%202018.pdf

Instituto de Estadística de la UNESCO (2017). Más de la mitad de los niños y adolescentes en el mundo no está aprendiendo. Ficha informativa 46. file:///H:/0\%20fs46-more-than-half-children-not-learning2017-sp.pdf
Mineduc.
(2017).
Enseñanza
de
la
comprensión
lectora.

http://www.usaidlea.org/images/Libro_Comprension_lectora_2017.pdf 
MINEDUC (2018). Educación en Ecuador. Resultados de PISA para el desarrollo. Instituto Nacional de Evaluación Educativa, Quito, Ecuador. http://evaluaciones.evaluacion.gob.ec/BI/informe-general-pisa2018/

Molina, L. E. (2016). Destrezas en la lectura comprensiva en los estudiantes de primero de bachillerato. (Tesis de maestría). Universidad Católica del Ecuador. https://repositorio.pucese.edu.ec/bitstream/123456789/833/1/MOLINA\%20CEDENO\%20LUZ\%20EST RELLITA.pdf

Muñoz, Á., And Ocaña, M. (2017). Use of meta-cognitive strategies for text comprehension. Cuadernos de Lingüística Hispánica (29), 223-244. https://doi.org/10.19053/0121053x.n29.2017.5865

Núñez, K., Medina, J. C., y González, J. (2019). Impacto de habilidades de comprensión lectora en el aprendizaje escolar: Un estudio realizado en una comuna de la región metropolitana, Chile. Revista Electrónica Educare, 23(2), 1-22. http://dx.doi.org/10.15359/ree.23-2.2

Pascual-Gómez, I., and Carril-Martínez, I. (2017). Relationship between Reading comprehension, spelling and academic performance. A study in Primary School students. Ocnos, unknown (2). https://doi.org/10.18239/ocnos

Parodi, G., Moreno, T., y Julio, C. (2020). Comprensión de textos escritos: reconceptualizaciones en torno a las demandas del siglo XXI. Íkala, 25(3), 775-795, https://doi.org/10.17533/udea.ikala.v25n03a10.

Ricaldi, J. (2019). Efectos de un programa de estrategias cognitivas en la comprensión lectora en estudiantes del segundo grado de educación secundaria de la Institución Educativa Politécnico Regional del Centro Huancayo. (Tesis de doctorado). Universidad Nacional de Educación Enrique Guzmán y Valle. https://alicia.concytec.gob.pe/vufind/Record/UNEI_0d96f0f8420f24f0d2313ea5a5c43266 